\title{
Slow-then-rapid quenching as traced by tentative evidence for enhanced metallicities of cluster galaxies at $z \sim 0.2$ in the slow quenching phase
}

\author{
C. Maier ${ }^{1}$, B. L. Ziegler ${ }^{1}$, C. P. Haines ${ }^{2}$, and G. P. Smith ${ }^{3}$ \\ ${ }^{1}$ University of Vienna, Department of Astrophysics, Tuerkenschanzstrasse 17, 1180 Vienna, Austria \\ e-mail: christian.maier@univie.ac.at \\ 2 INAF - Osservatorio Astronomico di Brera, Via Brera 28, 20121 Milano, Italy \\ 3 School of Physics and Astronomy, University of Birmingham, Edgbaston, Birmingham B15 2TT, UK
}

Received 20 September 2018 / Accepted 24 November 2018

\begin{abstract}
Aims. As large-scale structures in the Universe develop with time, environmental effects become more and more important as a star formation quenching mechanism. Since the effects of environmental quenching are more pronounced in denser structures that form at later times, we seek to constrain environmental quenching processes using cluster galaxies at $z<0.3$.

Methods. We explored seven clusters from the Local Cluster Substructure Survey (LoCuSS) at $0.15<z<0.26$ with spectra of 1965 cluster members in a mass-complete sample from the ACReS (Arizona Cluster Redshift Survey) Hectospec survey covering a region that corresponds to about three virial radii for each cluster. We measured fluxes of [O II] $\lambda 3727, \mathrm{H} \beta,[\mathrm{O} I I I] \lambda 5007, \mathrm{H} \alpha$, and [N II] $\lambda 6584$ emission lines of cluster members, enabling us to unambiguously derive $\mathrm{O} / \mathrm{H}$ gas metallicities. We also measured star formation rates (SFRs) from extinction-corrected $\mathrm{H} \alpha$ fluxes. We compared our cluster galaxy sample with a field sample of 705 galaxies at similar redshifts observed with Hectospec as part of the same survey.

Results. We find that star-forming cluster and field galaxies show similar median specific SFRs in a given mass bin of $1-3.2 \times 10^{10} M_{\odot}$ and 3.2-10 $\times 10^{10} M_{\odot}$, respectively. But their $\mathrm{O} / \mathrm{H}$ values are displaced, in the lower mass bin, to higher values (significance $2.4 \sigma$ ) at projected radii of $R<R_{200}$ compared with galaxies at larger radii and in the field. The comparison with metallicity-SFR-mass model predictions with inflowing gas indicates a slow-quenching scenario in which strangulation is initiated when galaxies pass $R \sim R_{200}$ by stopping the inflow of gas. We find tentative evidence that the metallicities of cluster members inside $R_{200}$ are thereby increasing, but their SFRs are hardly affected for a period of time because these galaxies consume available disk gas. We use the observed fraction of star-forming cluster galaxies as a function of clustercentric radius compared to predictions from the Millennium simulation to constrain quenching timescales to be $1-2 \mathrm{Gyr}$, which is defined as the time between the moment the galaxy passes $R_{200}$ until complete quenching of star formation. This is consistent with a slow-then-rapid quenching scenario. Slow quenching (strangulation) starts when the gas inflow is stopped when the galaxy passes $R_{200}$ with a phase in which cluster galaxies are still star forming, but they show elevated metallicities tracing the ongoing quenching. This phase lasts for $1-2 \mathrm{Gyr}$, and meanwhile the galaxies travel to denser inner regions of the cluster. This is followed by a "rapid" phase, i.e., a rapid complete quenching of star formation due to the increasing ram pressure toward the cluster center that can also strip the cold gas in massive galaxies.
\end{abstract}

Key words. galaxies: evolution - galaxies: clusters: general - galaxies: star formation - galaxies: abundances

\section{Introduction}

The internal properties of galaxies in dense environments are known to differ from isolated galaxies, as seen in their color (e.g., Peng et al. 2010), star formation rate (SFR; e.g., Wetzel et al. 2012), or morphology (e.g., Dressler 1980). A detailed understanding of the physics responsible for the differences between field and cluster galaxies has so far proved difficult, although a number of mechanisms have been proposed that could play a role in affecting and stopping (quenching) star formation in dense environments. A plausible cause of the quenching of star formation in galaxies after accretion into massive halos are their interactions with the dense intracluster medium (ICM) due to their high velocity passage through the dense ICM.

As a scenario for quenching satellite galaxies in groups and clusters, Wetzel et al. (2013) proposed a two-stage model in which star formation truncation happens rapidly, but only after a delay following accretion into the cluster. In this model, galaxies experience little or no change in their SFRs during the delay phase. The model has been used to successfully interpret a number of observations at $z<1$, but the hypothesis that galaxies are completely unaffected by environment during the delay period remains controversial and has been a theoretical challenge. It is difficult to understand and simulate galaxies traveling at $1000-2000 \mathrm{~km} \mathrm{~s}^{-1}$ through the cluster ICM, remaining uninfluenced by their environment for several gigayears, and then suddenly stopping the formation of stars on a short timescale. On the other hand, Haines et al. (2013) found the specific SFRs (SSFRs) of massive $\log \left(M / M_{\odot}\right)>10$ star-forming cluster galaxies within $R_{200}$ to be (slightly) lower than the SSFRs of their counterparts in the field, inferring that star-forming galaxies are slowly quenched upon accretion into clusters and are best fit by models in which their SFRs decline exponentially on quenching timescales in the range $0.7-2$ Gyr.

Several authors have studied the role of the SFR and of the SSFR $\left(\mathrm{SSFR}=\mathrm{SFR} / M_{*}\right)$ on the metallicity of a galaxy and 
presented links between SFR and metallicity (e.g., Ellison et al. 2008; Lara-López et al. 2010), finally claiming an epochindependent fundamental metallicity relation (FMR) between metallicity, mass and SFR, $Z\left(M_{*}, \mathrm{SFR}\right)$, expected to be applicable at all redshifts (Mannucci et al. 2010). In order to explain star formation as a second parameter in the mass-metallicity relation (MZR), some publications have ascribed an inflow of gas as the responsible driver for the dilution of metallicity and increase of SFR (Mannucci et al. 2010; Davé et al. 2012; Dayal et al. 2013). One explanation for the origin of this relation has been summarized by Lilly et al. (2013) and is known as the bathtub model. The SFR is thereby closely linked to the mass of gas in the galaxy modulated by infalling gas and outflows.

Peng et al. (2015) used the SDSS (Sloan Digital Sky Survey) local sample and found that the stellar metallicity of satellite galaxies is slightly higher than that of central galaxies. These authors inferred that lower mass satellite galaxies are more prone to strangulation, implying that environmental effects are responsible for stopping the inflow of gas. In Maier et al. (2016 MKZ16 in the following) we explored the $Z\left(M_{*}, \mathrm{SFR}\right)$ in the MACSJ0416.1-2403 cluster at $z \sim 0.4$ with a sample of mostly intermediate and low mass galaxies $\left(8.5<\log M / M_{\odot}<10.2\right)$, and only a handful of higher mass objects. We found a dependence of metallicity on enviroment at a given stellar mass that is manifested in higher metallicities in overdense regions, which we interpreted as a strangulation scenario. We call strangulation/starvation a scenario in which the supply of cold gas (through cooling) onto the galaxy disk is halted because the hot halo gas is stripped owing to external forces. In a context of a rich cluster, this scenario was first described by Larson et al. (1980), who used the removal of the gas reservoir because of external forces to explain the passive cluster galaxy population. For infalling cluster galaxies, the gas reservoir may be stripped or truncated as a consequence of interactions with cluster potential, ICM gas, or other galaxies (e.g., Bekki et al. 2002). This can terminate the fresh pristine gas accretion on to the galaxy (which would otherwise dilute the interstellar medium), increasing the retained gas metallicity before the galaxy completely stops forming stars, i.e., is completely "quenched", at a later time. Thus, the gas-phase metallicity can be used as a slow quenching (strangulation) tracer (cf. MKZ16).

In this paper we extend these investigations to a larger sample of cluster galaxies, measuring $\mathrm{O} / \mathrm{H}$ abundances in $K$-band selected galaxy samples in seven massive clusters at $0.15<z<$ 0.26 from LoCuSS. We contrast this sample to comparable measurements of field galaxies at similar redshifts observed at the same time and with the same Hectospec instrument configuration as the cluster galaxies. We do this using the two connected relations, the MZR and the mass- $(\mathrm{S}) \mathrm{SFR}$ relation, studying the environmental dependence of these relations and the implications for the quenching processes.

To estimate the chemical abundances, a number of diagnostics have been developed based on strong emission lines (ELs), [O II $] \lambda 3727, \mathrm{H} \beta,[\mathrm{O} \mathrm{III}] \lambda 5007, \mathrm{H} \alpha$ and [N II] $\lambda$ 6584. At higher redshifts, these ELs move to the near-infrared, and $\mathrm{H} \alpha$ and [N II] $\lambda 6584$ are already shifting beyond optical above redshifts of $\sim 0.5$, requiring near-infrared spectroscopy to observe these ELs (e.g., Maier et al. 2014, 2015). Thus, the $0.15<z<0.26$ epoch we probe is a redshift range at which all these ELs can be observed free of strong night skylines and with the same optical spectrograph (in our case Hectospec), without the complication of obtaining a good relative calibration between spectra from optical and near-infrared spectrographs.
In MKZ16 we studied star-forming galaxies in one cluster and found indications that accreted cluster star-forming galaxies have similar SFRs to their field counterparts, but their gas-phase metallicities are enhanced owing to an ongoing strangulation (slow quenching) process. In this study we reinforce the findings of MKZ16 by studying seven clusters and further extend the discussion of a plausible strangulation scenario, now in a slow-then-rapid framework. Stopping the inflow of gas hardly affects the SFRs of cluster galaxies during a slow-quenching phase, but enhances the gas-phase metallicities of cluster galaxies compared to field counterparts, before the star formation is (rapidly) completely quenched at a later time.

The paper is structured as follows: in Sect. 2 we present the selection of the cluster EL galaxies at $z \sim 0.2$ and their Hectospec spectroscopy. We investigate the active galactic nucleus (AGN) contribution and present the derivation of SFRs, metallicities, and stellar masses of the cluster and field galaxies we observed. In Sect. 3 we present the phase-space diagram, the MZR, and the mass-SSFR relations at $z \sim 0.2$, and investigate their dependence on environment as a function of the clustercentric radius of the clusters as well. We discuss how the cluster environment affects the chemical enrichment. In Sect. 4 we compare our observed mass-metallicity-SFR values with bathtub model predictions with inflow of pristine gas, finding indications of a strangulation scenario similar to the findings of MKZ16: the inflow of pristine gas is halted and strangulation is initiated when galaxies are accreted inside $R_{200}$ of the cluster. We compare the position of galaxies in the phase-space diagram with theoretical predictions in order to constrain the time between infall into the cluster and complete quenching of star formation (quenching timescales). We thereby compare the observed radial population gradients of star-forming galaxies with predictions from the Millennium simulation (Springel et al. 2005). We then discuss the slow-then-rapid quenching scenario implied by our findings. Finally in Sect. 5 we summarize our conclusions. A concordance cosmology with $H_{0}=70 \mathrm{~km} \mathrm{~s}^{-1} \mathrm{Mpc}^{-1}, \Omega_{0}=0.25, \Omega_{\Lambda}=0.75$ is used throughout this paper. We assume a Salpeter (Salpeter 1955) initial mass function (IMF) for all derived stellar masses and SFRs and correct existing measurements used in this paper to a Salpeter IMF. We note that metallicity and abundance are taken to denote oxygen abundance, $\mathrm{O} / \mathrm{H}$, throughout this paper, unless otherwise specified. In addition, we use dex throughout to denote the antilogarithm, i.e., $0.3 \mathrm{dex}$ is a factor of two.

\section{Data and measurements}

\subsection{Cluster galaxies from LoCuSS and sample selections}

The LoCuSS (Local Cluster Substructure Survey) is a multiwavelength survey of X-ray luminous galaxy clusters at $0.15<$ $z<0.3$ (Smith et al. 2010) drawn from the ROSAT All Sky Survey cluster catalog. These clusters benefit from a rich data set, including Subaru/Suprime-Cam optical imaging, Spitzer/MIPS $24 \mu \mathrm{m}$ maps, Herschel/PACS+SPIRE 100-500 $\mu \mathrm{m}$ maps, Chandra and XMM X-ray data, GALEX UV data, and near-infrared imaging. All of this data cover at least $25 \times 25$ square arcminute fields centered on the clusters.

The ACReS (Arizona Cluster Redshift Survey) is a large spectroscopic survey using Hectospec/MMT of 30 massive clusters of LoCuSS, seven of which we study in this paper (see Table 1). The 270 line grating was used, providing a wide wavelength range (3650-9200 $\mathrm{A}$ ) at $6.2 \AA$ resolution. The main strengths of ACReS are its wide field of view (FoV; $\sim 1^{\circ}$ diameter), which reaches well into the infall regions (up to about 
Table 1. Seven clusters of this study.

\begin{tabular}{ccccccccc}
\hline \hline $\begin{array}{c}\text { Cluster } \\
\text { name }\end{array}$ & $z$ & RA & Dec & $\begin{array}{c}R_{200} \\
(\mathrm{Mpc})\end{array}$ & $\begin{array}{c}M_{200} \\
\left(10^{14} M_{\odot}\right)\end{array}$ & $\begin{array}{c}\text { Cluster } \\
\text { members }^{a}\end{array}$ & $\begin{array}{c}\text { SF cluster }_{\text {members }}{ }^{a} \\
\text { members }^{\text {Mith O/H }}{ }^{a}\end{array}$ \\
\hline RXJ1720 & 0.1599 & $17: 20: 10.14$ & $+26: 37: 30.90$ & 2.02 & 9.40 & $358 / 219$ & $63 / 22$ & $45 / 15$ \\
A1914 & 0.1671 & $14: 25: 59.78$ & $+37: 49: 29.10$ & 1.90 & 7.69 & $388 / 276$ & $101 / 50$ & $65 / 31$ \\
A1689 & 0.1851 & $13: 11: 29.45$ & $-01: 20: 28.30$ & 2.13 & 11.01 & $438 / 291$ & $116 / 46$ & $66 / 32$ \\
A963 & 0.2043 & $10: 17: 01.20$ & $+39: 01: 44.40$ & 1.81 & 6.75 & $414 / 332$ & $107 / 75$ & $69 / 45$ \\
A2390 & 0.2291 & $21: 53: 36.72$ & $+17: 41: 31.20$ & 2.21 & 12.36 & $371 / 287$ & $86 / 70$ & $61 / 48$ \\
A1763 & 0.2323 & $13: 35: 16.32$ & $+40: 59: 45.60$ & 1.85 & 7.18 & $292 / 230$ & $76 / 63$ & $53 / 43$ \\
A1835 & 0.2520 & $14: 01: 02.40$ & $+02: 52: 55.20$ & 2.27 & 13.34 & $465 / 330$ & $112 / 84$ & $62 / 45$ \\
\hline
\end{tabular}

Notes. The table lists: mean redshift of cluster members, coordinates, radii $R_{200}$ and cluster masses $M_{200}$ (as reported in Haines et al. 2013); number of cluster members, star-forming (SF) objects and galaxies with $\mathrm{O} / \mathrm{H}$ measured in our sample. The "200" subscript refers to the radius where the average density is 200 times the critical density of the Universe at the cluster redshift. ${ }^{(a)}$ The second number is the number of galaxies with masses above the completeness limit, with $10 \leq \log \left(M / M_{\odot}\right) \leq 11$.

three virial radii) of the clusters, and the careful target selection based on $J$ and $K$ near-infrared imaging, which provides an unbiased, mass-complete sample of cluster galaxies, down to $\log \left(M / M_{\odot}\right) \sim 10$ (cf. Haines et al. 2015). We note that, while the stellar masses derived by Haines et al. (2015) used an older relation of Bell et al. (2003), we now use a newer relation from Zibetti et al. (2009) to derive stellar masses, as described in Sect. 2.3, and get a slightly different mass-completness limit of $\log \left(M / M_{\odot}\right) \sim 10$.

Members of each cluster were identified from the redshift versus projected clustercentric radius plot as lying within the "trumpet" -shaped caustic profile expected for galaxies infalling and subsequently orbiting within a massive virialized structure (see details in Haines et al. 2013, 2015). A field sample was carefully determined to be in narrow redshift slices on either side of the clusters. Because the properties of galaxies in the infall regions of the clusters are known to be systematically different from the field even at 3-4 $R_{200}$ (e.g., Wetzel et al. 2012), we excluded from the field sample all galaxies within $4000 \mathrm{~km} \mathrm{~s}^{-1}$ of the cluster (see more detail in Haines et al. 2013).

The selection of 7 out of 30 clusters was performed based on following criteria. First, we excluded 6 clusters with $z>0.26$; [NII] EL was redshifted out of the observed spectrum range. We did not use 4 additional clusters without SDSS photometry. An additional 13 clusters were excluded because of their much lower number $(<200)$ of total spectroscopic members compared to the typical about 300-400 (or even >400) spectroscopic members per cluster of this study (Table 1). A small number of total spectroscopic members corresponds to a significant reduction of the number, in the mass-complete sample, of cluster members with $\mathrm{O} / \mathrm{H}$ measurements (OHgals sample, see last column of Table 1). To avoid any bias due to such a small number of OHgals objects per cluster, we excluded these 13 clusters with $<200$ spectroscopic members from the present study.

\subsection{Star-forming galaxies and Type-2 AGNs}

Cid Fernandes et al. (2011) introduced the WHAN diagram, $E W_{\mathrm{H} \alpha}$ (equivalent width of $\mathrm{H} \alpha$ ) versus $[\mathrm{NII}] / \mathrm{H} \alpha$, and used it to provide a comprehensive emission-line classification of SDSS galaxies. This diagram is useful to distinguish Type-2 AGNs from star-forming galaxies with weak [OIII] and $\mathrm{H} \beta$ ELs. We used the WHAN diagram and followed the $\log ([\mathrm{NII}] / \mathrm{H} \alpha)$ criterium of Gordon et al. (2018), who used the WHAN diagram to identify Type-2 AGNs for the GAMA (Galaxy and Mass Assembly) survey. Specifically, for our mass-complete cluster galaxy sample, we classified as SFgals (star-forming galaxies) 410 star-forming galaxies with a rest-frame $E W(\mathrm{H} \alpha)>3 \AA$, a $S / N>5$ in $\mathrm{H} \alpha$ EL flux and $\log ([\mathrm{NII}] / \mathrm{H} \alpha) \leq-0.3$, and as SeyfLiners (Type-2 AGNs) 215 objects with a rest-frame $E W(\mathrm{H} \alpha)>3 \AA$, a $S / N>5$ in $\mathrm{H} \alpha$ EL flux and $\log ([\mathrm{NII}] / \mathrm{H} \alpha)>$ -0.3 (see Fig. 1). The remaining 1340 cluster galaxies belong to the mass-complete nonSFgals (non-star-forming galaxies) sample.

The selection of the SFgals sample is carried out using the WHAN diagram to ensure a complete sample of star-forming galaxies for comparison of the fraction of star-forming galaxies with theoretical models and simulations, including galaxies with stronger $\mathrm{H} \alpha$, but weak [OIII] or $\mathrm{H} \beta$. On the other hand, we use the BPT diagram to select a sample OHgals of galaxies with high enough signal-to-noise ratio in [OIII] and $\mathrm{H} \beta$ to derive reliable metallicities. This is done as follows. We first required a restframe $E W(\mathrm{H} \alpha)>3 \AA$, a $S / N>5$ in $\mathrm{H} \alpha$ EL flux, a $S / N>2$ in each $\mathrm{H} \beta$, [OIII] and [NII] EL fluxes, and a rest-frame $E W(\mathrm{H} \beta)>$ $2 \AA$. From this sample we then excluded composite/AGN galaxies following the BPT diagram shown in Fig. 2. Taking error bars of the flux measurements into account, and to mimimize the number of excluded composite galaxies above but close to the demarcation line that are likely to have their $\mathrm{H} \alpha$ line mostly produced by star formation, we also include in the OHgals sample a few objects above and up to a maximum of 0.3 dex to the right of the Kauffmann et al. (2003) curve. We are left with a sample of 259 galaxies in the mass-complete OHgals cluster sample and 169 galaxies in the mass-complete OHgals field sample. The numbers of OHgals and composite/AGN galaxies (CompAGNs) in the different mass bins for the field and cluster samples are given in Table 2.

\subsection{Oxygen abundances, SFRs, and stellar masses}

After reduction of the Hectospec/MMT data using the SPECROAD pipeline (Mink et al. 2007), the EL fluxes of [O II] $\lambda 3727, \mathrm{H} \beta$, [O III] $\lambda 5007, \mathrm{H} \alpha$, and [N II] $\lambda 6584$ for cluster member and field galaxies were measured (including stellar absorption correction for $\mathrm{H} \alpha$ and $\mathrm{H} \beta$ ) as described in detail in M. J. Pereira et al. (in prep., see also Pereira et al. 2012). As in MKZ16, the Eq. (4) in Kewley et al. (2013), $12+\log (\mathrm{O} / \mathrm{H})=8.97-0.32 \times \log (([\mathrm{OIII}] / \mathrm{H} \beta) /([\mathrm{NII}] / \mathrm{H} \alpha))$, which 


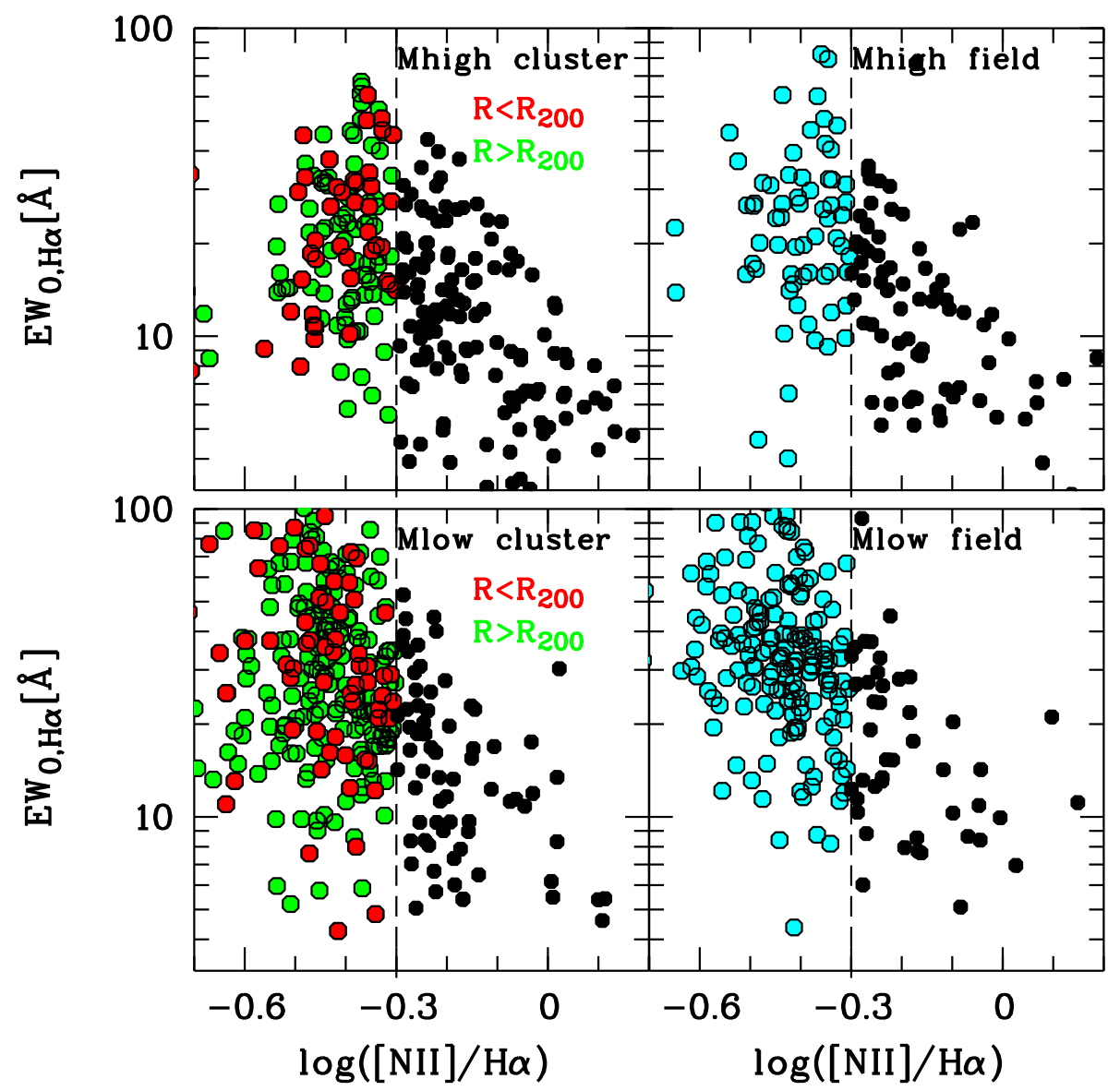

Fig. 1. Diagnostic diagram WHAN (Cid Fernandes et al. 2011) to distinguish star formation-dominated galaxies from AGNs for the LoCuSS mass-complete cluster and field galaxy samples. The star-forming region of the WHAN diagram is populated by 410 cluster galaxies and 227 field galaxies (SFgals sample, red, green, and cyan points) with rest-frames $E W(\mathrm{H} \alpha)>3 \AA$ and $\log ([\mathrm{NII}] / \mathrm{H} \alpha) \leq-0.3$. The red symbols show the subsample of SFgals with $R<R_{200}$. Mlow (Mhigh) galaxies shown as black symbols in the lower (upper) panels lie in the region occupied by Type-2 AGNs (SeyfLiners sample, see Table 2). corresponds to the Pettini \& Pagel (2004) O3N2 calibration for the Kewley \& Dopita (2002) metallicity scale, was used to consistently compute metallicities for the cluster and field galaxies, and for the comparison SDSS sample. The SDSS sample was selected and matched as described in MKZ16 to ensure a consistent comparison of the physical properties of the SDSS and OHgals samples.

One of the most reliable and well-calibrated SFR indicators is the $\mathrm{H} \alpha \mathrm{EL}$ (Kennicutt 1998). The conversion of $\mathrm{H} \alpha$ luminosity to SFR requires several steps. To correct for fiber losses for cluster galaxies we convolved each Hectospec spectrum with the SDSS $r$-band filter and then compared this magnitude with the SDSS $r$-band magnitude of the respective galaxy. The difference between the two magnitudes gave the aperture correction factor for each spectrum. This correction assumes that the $\mathrm{H} \alpha$ line flux and the $r$-band continuum suffer equal slit losses. Thus revised $\mathrm{H} \alpha$ line luminosities $L(\mathrm{H} \alpha)$ were corrected for extinction based on the Balmer decrement and then transformed into SFRs by applying the Kennicutt (1998) conversion, SFR $\left(M_{\odot} \mathrm{yr}^{-1}\right)=7.9 \times 10^{-42} \mathrm{~L}(\mathrm{H} \alpha) \mathrm{ergs} \mathrm{s}^{-1}$.

Stellar masses were computed from the available photometry using the $g-i$ color-dependent $K$-band mass-to-light relation $\log (M / L)_{K}=-1.321+0.754 \cdot(g-i)$ reported by Zibetti et al. (2009). We converted the masses from a Chabrier (2003) IMF to a Salpeter (1955) IMF (as used in this paper) by multiplying the Chabrier (2003) IMF masses by 1.7. This factor of 1.7 was found by Pozzetti et al. (2007) to be a systematic median offset, with a very small dispersion, in the masses derived with the two different IMFs; this value is rather constant for a wide range of star formation histories.

\section{Results: Environmental effects on the metallicities and SFRs of cluster galaxies}

The phase-space diagram (projected clustercentric radius versus line-of-sight velocity relative to the cluster redshift) provides valuable information on the accretion history of cluster galaxies (e.g., Haines et al. 2015). Figure 3 shows the phase-space diagram for the seven clusters, where $\mathrm{R}$ is the projected radial distance from the cluster center; the different symbols indicate different galaxy subsamples, i.e., nonSFgals (cyan), SFgals (blue), and OHgals (magenta). We note that the OHgals sample is a subset of the star-forming sample, SFgals. The histograms on the right with the number of objects as a function of clustercentric radius in different subsamples are later used (in Sect. 4.3) to compute the fraction of star-forming galaxies and to derive quenching timescales.

As shown in Fig. 13 of Haines et al. (2015), infalling galaxies that have recently passed within $R_{200}$ for the first time, but have yet to reach the pericenter, have high line-of-sight velocities, which are accelerated to high speeds as the galaxies fall deep into the gravitational potential well of the cluster core. The star-forming galaxies at $R<R_{200}$ (magenta and blue symbols) shown in the phase-space diagram (Fig. 3) have in general higher line-of-sight velocities than non-star-forming galaxies (cyan filled circles); this is indicative that these galaxies are an infalling population.

For a meaningful investigation and considering our sample of targets (e.g., only seven cluster galaxies in the OHgals sample have $\left.\log \left(M / M_{\odot}\right)>11\right)$, we divide the galaxies in our masscomplete sample into two mass bins: i) galaxies with Mlow 


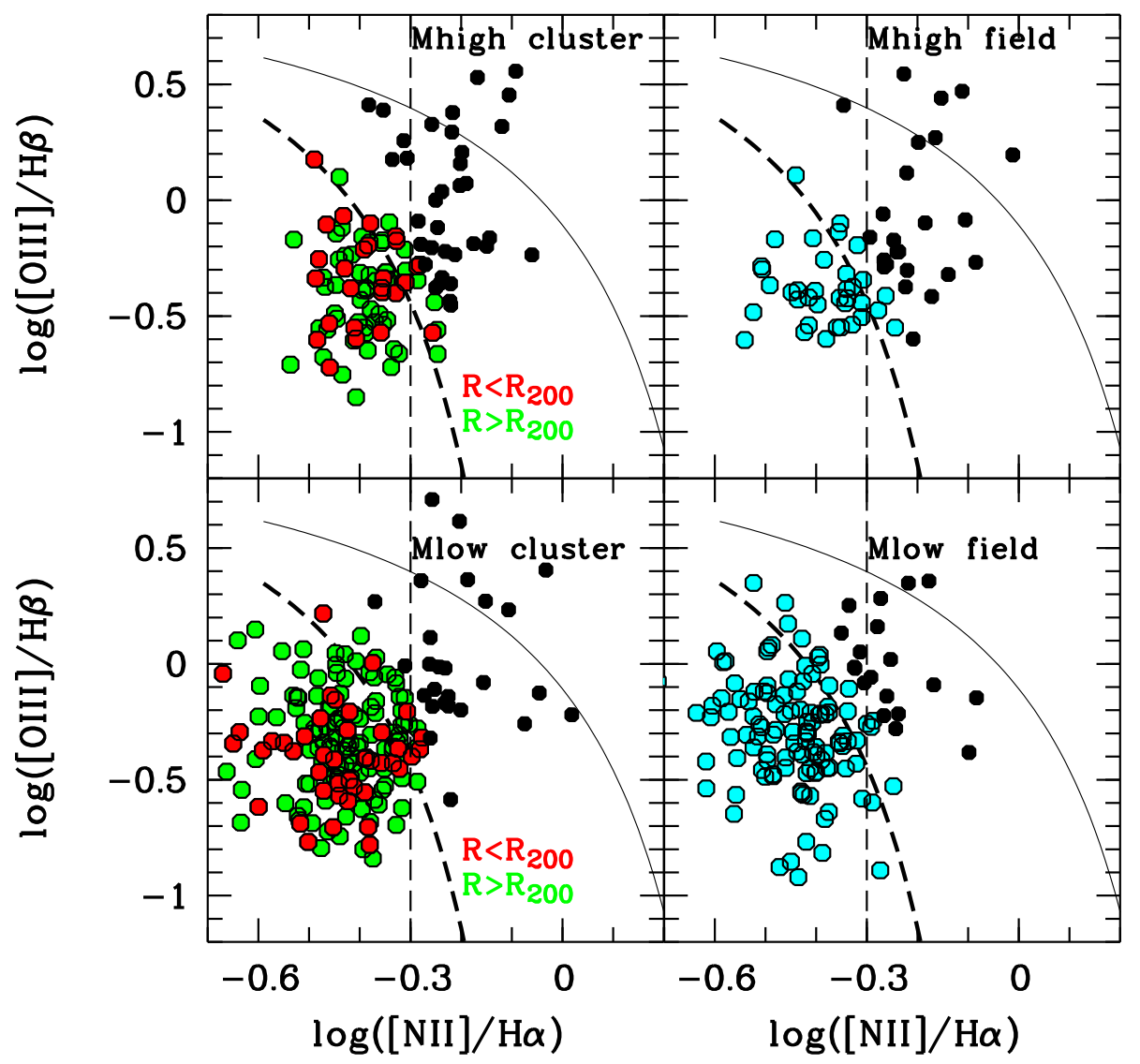

Fig. 2. Diagnostic diagram BPT (Baldwin et al. 1981) to distinguish star formation-dominated galaxies from AGNs for the mass-complete cluster and field galaxy samples. The star-forming region of the BPT diagram has 259 cluster galaxies and 169 field galaxies (OHgals sample, red, green and cyan points), lying under and to the left of the empirical (dashed) curve of Kauffmann et al. (2003). We also include in the OHgals sample a few objects above and up to a maximum of 0.3 dex to the right of the Kauffmann et al. (2003) curve to take error bars of the flux measurements into account and to mimimize the number of excluded composite galaxies above but close to the demarcation line that are likely to have their $\mathrm{H} \alpha$ line mostly produced by star formation. The red symbols show the subsample of OHgals with $R<R_{200}$. Mlow (Mhigh) galaxies shown as black symbols in the lower (upper) panels lie in the region occupied by AGN and composite galaxies (CompAGNs sample, see Table 2), with composites lying between the empirical dashed curve of Kauffmann et al. (2003) and the theoretical solid curve of Kewley (2001). The vertical dashed line at $\log ([\mathrm{NII}] / / \mathrm{H} \alpha)=-0.3$ shows the demarcation line for selecting SeyfLiners galaxies $(\log ([\mathrm{NII}] / \mathrm{H} \alpha)>-0.3)$ using the criterium of Cid Fernandes et al. (2011), see Fig. 1.

Table 2. Number of objects in different subsamples of cluster and field galaxies.

\begin{tabular}{ccccccc}
\hline \hline Cluster sample & & & & & \\
& Parent sample & SFgals & nonSFgals & SeyfLiners & CompAGNs & OHgals \\
\hline Spectroscopic redshifts & 2726 & 661 & 1792 & 273 & 87 & 421 \\
$10 \leq \log \left(M / M_{\odot}\right)<10.5$ & 943 & 269 & 599 & 75 & 29 & 174 \\
$10.5 \leq \log \left(M / M_{\odot}\right) \leq 11$ & 1022 & 141 & 741 & 140 & 39 & 85 \\
$10 \leq \log \left(M / M_{\odot}\right) \leq 11$ & 1965 & 410 & 1340 & 215 & 68 & 259 \\
\hline Field sample & & & & & & \\
\hline Spectroscopic redshifts & 1068 & 443 & 452 & 173 & 66 & 284 \\
$10 \leq \log \left(M / M_{\odot}\right)<10.5$ & 307 & 146 & 106 & 55 & 24 & 125 \\
$10.5 \leq \log \left(M / M_{\odot}\right) \leq 11$ & 398 & 81 & 232 & 85 & 29 & 44 \\
$10 \leq \log \left(M / M_{\odot}\right) \leq 11$ & 705 & 227 & 338 & 140 & 53 & 169 \\
\hline
\end{tabular}

Notes. See Sect. 2.2 for details of the selection of the subsamples.

$\left(10 \leq \log \left(M / M_{\odot}\right)<10.5\right)$ and ii) galaxies with Mhigh $(10.5 \leq$ $\left.\log \left(M / M_{\odot}\right) \leq 11\right)$.

\subsection{Mass-SSFR relation in field and clusters at $z \sim 0.2$}

Peng et al. (2010) derived a formula of the evolution of the SSFR as a function of mass and time that we use to calculate the mean SSFR as a function of stellar mass at $z \sim 0.2$. For this, we assume a dependence of the SSFR on mass as observed for local SDSS galaxies and revised by Renzini \& Peng (2015), SSFR $\propto M^{\beta}$ with $\beta=-0.24$ (Fig. 4, oblique solid black line). The local main sequence (MS) relation was found to have a dispersion of a factor of 0.3 dex about the mean relation (e.g., Salim et al. 2007; Elbaz et al. 2007; Peng et al. 2010), indicated 

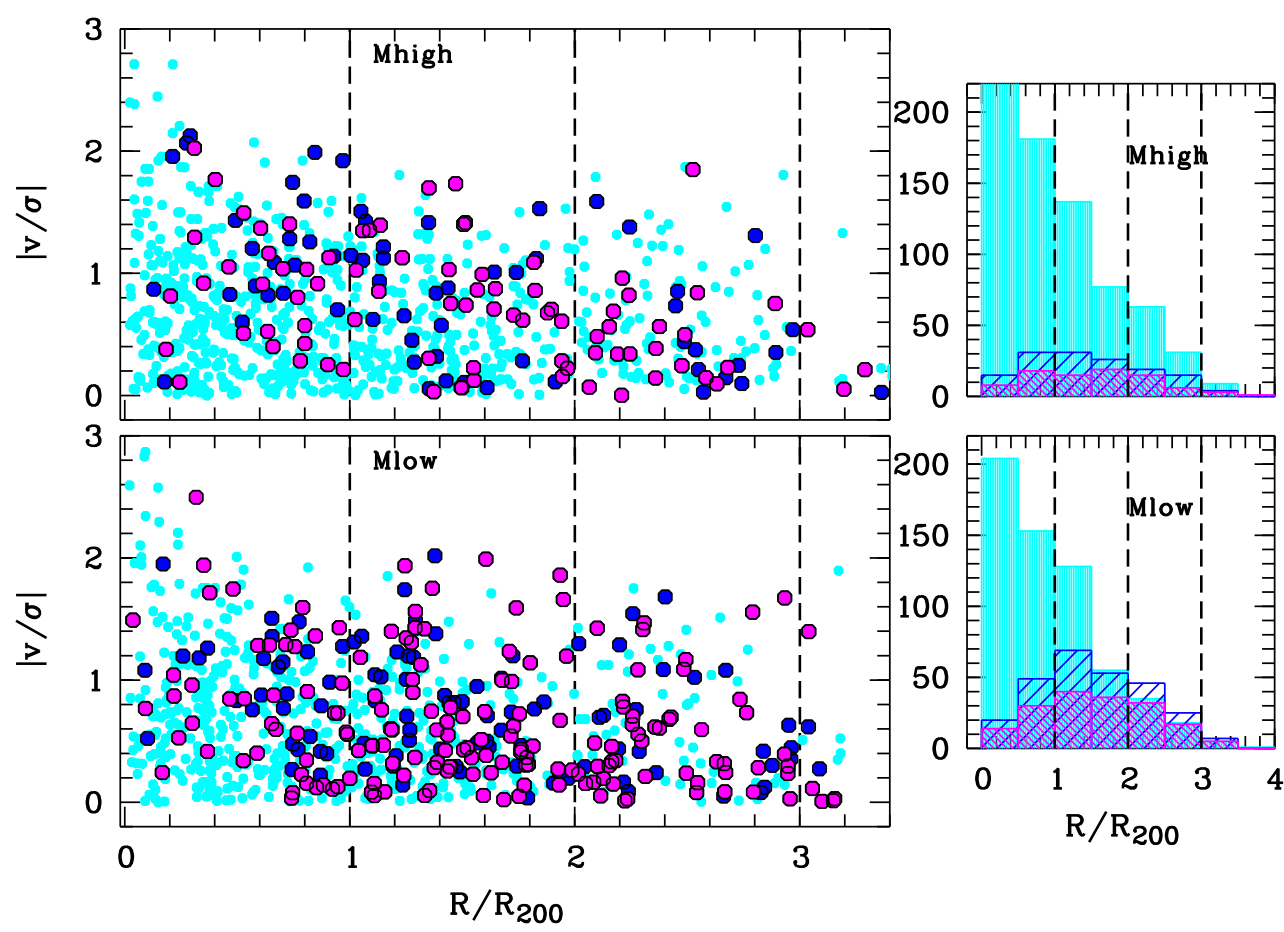

Fig. 3. Phase-space diagram showing cluster SFgals (blue), nonSFgals (cyan), and OHgals (magenta) objects with $10 \leq \log \left(M / M_{\odot}\right) \leq 11$. The histograms on the right for the different subsamples as a function of clustercentric radius are used to compute the observed fraction of star-forming galaxies and constrain quenching timescales by comparing with the Millennium simulation (cf. Fig. 7). The star-forming galaxies at $R<R_{200}$ (magenta and blue symbols) have in general higher line-of-sight velocities than non-starforming galaxies; this is indicative that these galaxies are an infalling population.

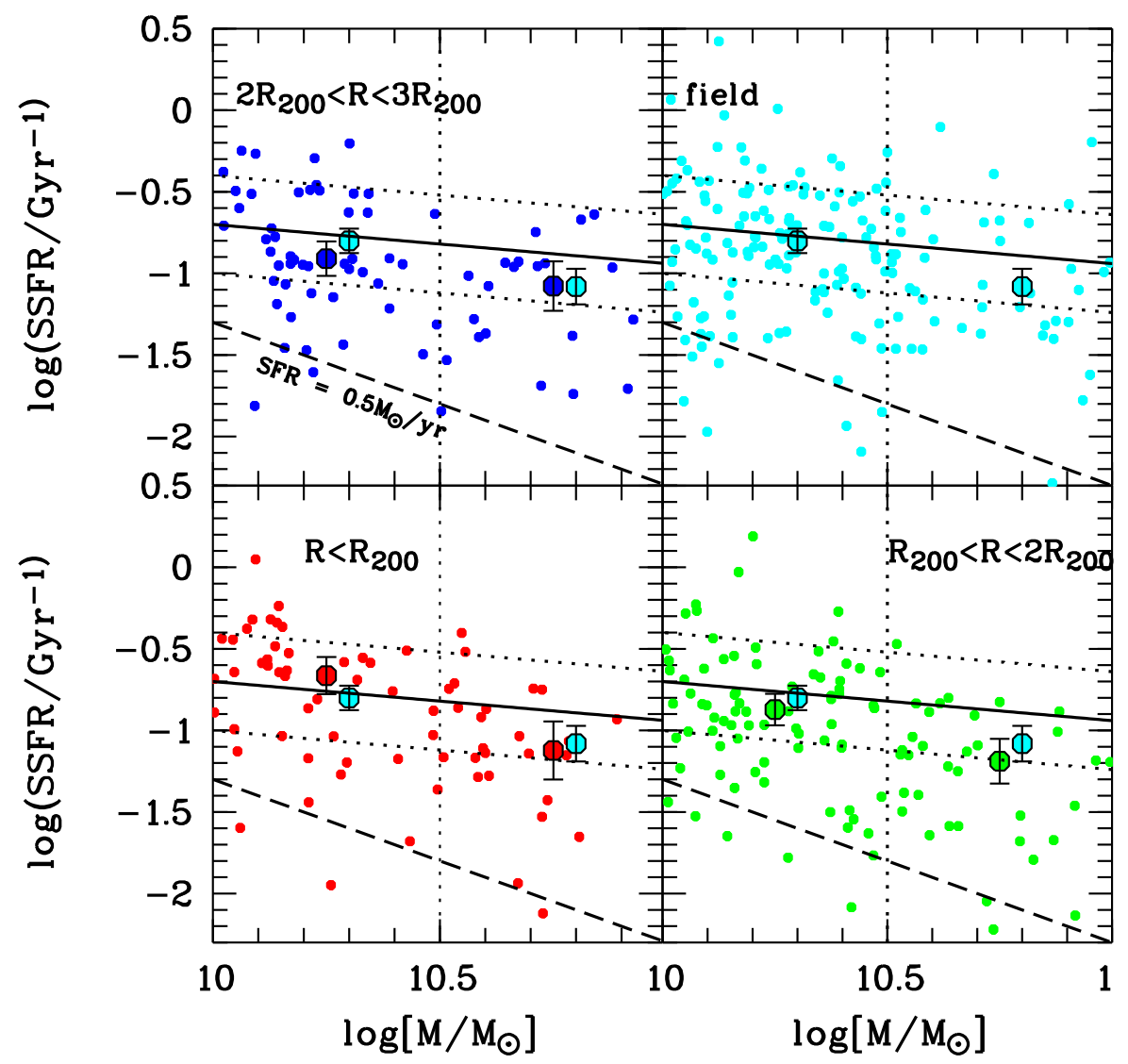

Fig. 4. Mass-SSFR relation for 259 cluster and 169 field galaxies in the OHgals mass-complete sample for three regions at different distances from the cluster center (red, green, and blue) and field galaxies (cyan). The oblique solid line shows the MS at $z \sim 0.2$ and its dispersion (indicated by the dotted lines) using Eq. (1) in Peng et al. (2010). The dashed oblique line in each panel shows that galaxies in our OHgals sample have typically SFRs $>0.5 M_{\odot} \mathrm{yr}^{-1}$. The median SSFRs in the Mhigh and in the Mlow mass bins are shown as big filled circles, with the field median values (cyan symbols) shown in all panels (slightly displaced in mass compared to cluster galaxies for visibility of the median symbols). In a given mass bin the median SSFR values of field and cluster galaxies are consistent. by the dotted oblique lines around the mean MS relation at $z \sim 0.2$ in Fig. 4 .

In a given mass bin (Mlow or Mhigh) the median SSFR values of field and cluster galaxies are consistent, given their error bars. Thus, the mass-SSFR relation of star-forming galaxies in our OHgals sample is rather independent of whether the galaxies are field or cluster galaxies and independent of clustercentric radius. This is consistent with the MKZ16 results studying the
MACSJ0416.1-2403 cluster and finding a similar distribution of star-forming cluster and field galaxies in the SSFR-mass diagrams.

\subsection{Enhanced metallicities of cluster galaxies inside $R_{200}$}

Figure 5 shows the MZR of the $z \sim 0.2$ cluster and field galaxies. All metallicities were computed with the same method, 

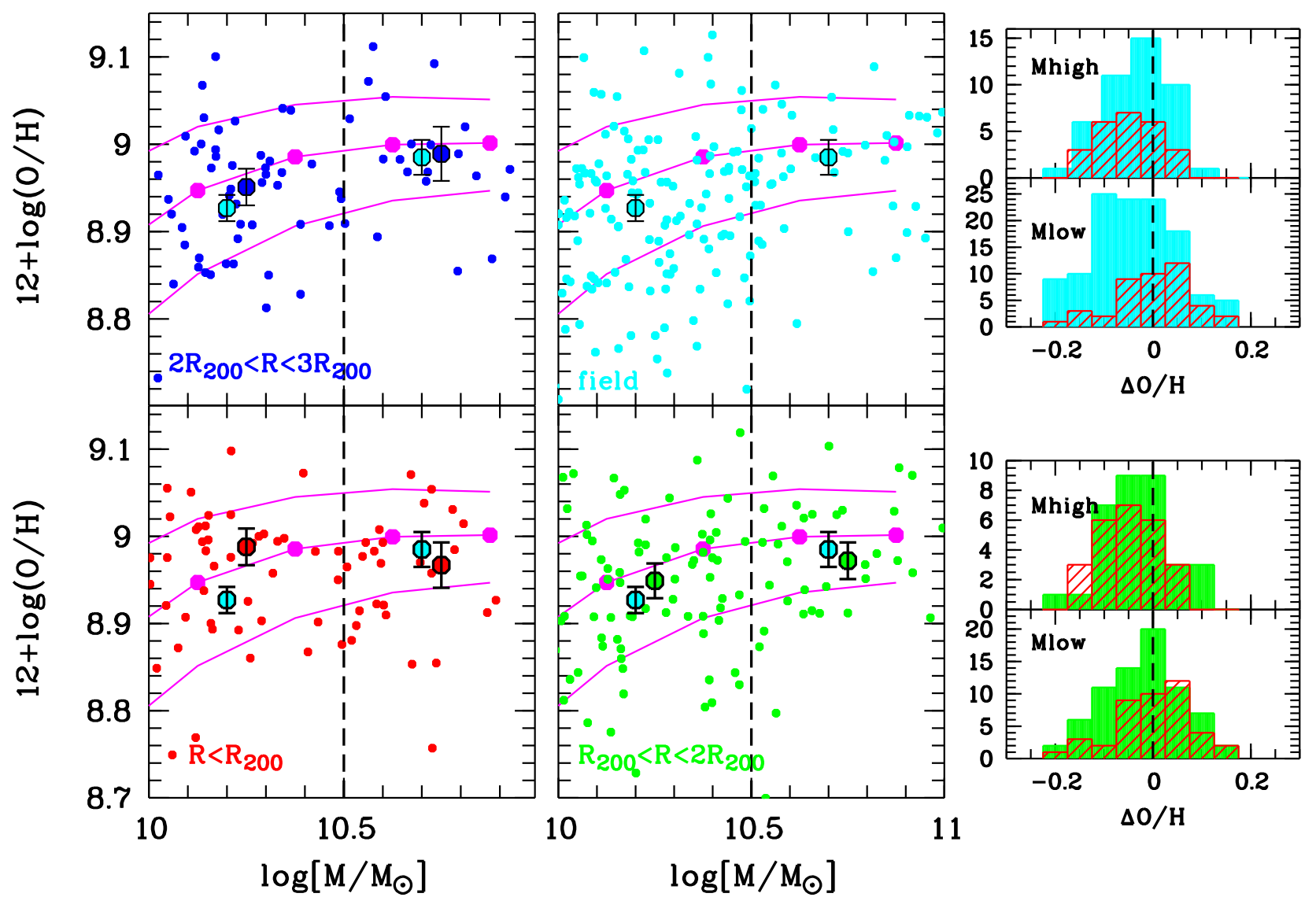

Fig. 5. MZR and dependence on environment of the 259 cluster galaxies and 169 field galaxies of the mass-complete OHgals sample. For the matched SDSS sample, we indicate 16th and 84th percentiles, and the medians (50th percentiles) of the distribution of $\mathrm{O} / \mathrm{H}$ values in the respective mass bin as magenta lines and symbols. The median $\mathrm{O} / \mathrm{Hs}$ in the mass-complete Mhigh and Mlow mass bins are shown as big filled circles; the field median values (cyan symbols) are shown in all panels (slightly displaced in mass compared to cluster galaxies for visibility of the median symbols). The field median $\mathrm{O} / \mathrm{H}$ value of Mhigh galaxies of the LoCuSS sample is similar to the SDSS median value at higher masses, while the Mlow field median value is slighty lower than the Mlow SDSS median value. The field Mlow and Mhigh median metallicities values are comparable to the respective (Mlow, Mhigh) cluster $\mathrm{O} / \mathrm{H}$ medians for $R>R_{200}$. On the other hand, the median $\mathrm{O} / \mathrm{H}$ value of Mlow galaxies in the more central part of the clusters $\left(R<R_{200}\right)$ is higher than the median $\mathrm{O} / \mathrm{H}$ field value, by about 0.06 dex, with a $2.4 \sigma$ significance. The histograms on the right showing the offsets from the local SDSS MZR relation, $\Delta O / H$, also clearly reveal enhanced metallicities for Mlow cluster galaxies inside $R_{200}$ (red histograms) compared to field galaxies and galaxies at $R>R_{200}$. This indicates that the cluster environment has the effect of increasing the gas metallicities of Mlow galaxies inside $R_{200}$ compared to their field counterparts of similar masses.

as described in Sect. 2.3. The field median Mlow and Mhigh metallicities (cyan symbols) are similar to the $R>R_{200}$ cluster median metallicity at a given mass (Mlow or Mhigh, respectively). On the other hand, the median metallicity of Mlow galaxies in the higher density parts of the clusters ( $R<R_{200}$, red symbol) is higher than the median metallicity of Mlow field galaxies, by about $0.06 \mathrm{dex}$, with a $2.4 \sigma$ significance.

The distribution of the metallicities of $R<R_{200}$ Mlow galaxies (small red filled symbols) is clearly shifted to higher values compared to their field counterparts (small cyan symbols). This is also seen in the histograms of the $\mathrm{O} / \mathrm{H}$ offsets from the local SDSS MZR relation $\Delta \mathrm{O} / \mathrm{H}$ (shown on the right in Fig. 5), which clearly depict enhanced metallicities of Mlow cluster galaxies inside $R_{200}$ (red histogram) compared to field galaxies (cyan histogram) and to galaxies at $R>R_{200}$ (green histogram). At higher masses, Mhigh, the metallicities of cluster and field galaxies broadly agree, with no sign of strong environmental effects on the $\mathrm{O} / \mathrm{H}$ values. In the next section, we discuss the reason for this behavior at Mhigh, where the MZR saturates.

\section{Discussion: strangulation and slow-then-rapid quenching}

\subsection{Strangulation implied by the comparison to the $Z\left(M_{*}, S F R\right)$ expectations with inflow of pristine gas}

To further explore the reason for the enhancement of $\mathrm{O} / \mathrm{Hs}$ for Mlow galaxies in the cluster environment we calculate the expected $\mathrm{O} / \mathrm{H}$ values from the Lilly et al. (2013) model for each galaxy individually with their respective stellar mass $M_{*}$ and SFR. Lilly et al. (2013) proposed a simple model of galaxy evolution in which the SFR is regulated by the mass of gas present in a galaxy, implying that $\mathrm{Z}$ depends on both $M_{*}$ and current SFR. Their derived $Z\left(M_{*}, \mathrm{SFR}\right)$ relation has a particular analytic form: the equilibrium metallicity $Z_{\mathrm{eq}}$ in the gas regulator model is given by $Z_{\mathrm{eq}}=Z_{0}+f_{\text {star }} y$, where $Z_{0}$ is the metallicity of the infalling gas and $y$ the yield. The quantity $f_{\text {star }}=S F R / \Phi$ is the fraction of the incoming baryons (inflow $\Phi$ ) that are converted into long-lived stars. We use their derived $Z\left(M_{*}, \mathrm{SFR}\right)$ relation assuming primordial inflowing gas $\left(Z_{0}=0\right)$ in their regulator bathtub model. We then compare the predicted values with the measured 
values and show the difference in Fig. 6. Studies of blue supergiants (Bresolin et al. 2016) and red supergiants (Davies et al. 2017) showed that the strong-line calibration yielding the most accurate (absolute) metallicities is the O3N2 calibration that we use. Therefore, our measured O3N2 metallicities should be well suited to be compared, in an absolute way, with the metallicities derived using the Lilly et al. (2013) model.

The median values depicted in Fig. 6 show that Mlow cluster galaxies inside $R_{200}$ (red) have higher metallicities compared to the Lilly et al. (2013) predictions with inflow of pristine gas, while Mlow field galaxies and cluster galaxies at $R>R_{200}$ are in general consistent with these predictions as shown by their median values. This indicates a strangulation scenario such as that discussed by MKZ16 (by studying the cluster MACSJ0416.1-2403). If the SFR is hardly affected (as discussed in Sect. 3.1), a higher value for the metallicity $Z_{\mathrm{eq}}$ in the above equation can be only obtained by decreasing or stopping the inflow of pristine gas $\Phi$ (in the denominator in the above equation). The inflow of pristine gas onto the galaxy disk is likely halted when Mlow cluster galaxies are accreted inside $R_{200}$, and their gas-phase metallicities thus increase because their interstellar medium is no longer diluted by the inflow of pristine gas.

The MZR saturates when the mass of oxygen produced by massive stars (which goes into the interstellar medium) equals the mass of oxygen locked up by lower mass stars that live longer. For Mhigh in the LoCuSS sample, the MZR saturates and becomes flat for higher masses, as seen in Fig. 5 for all environments (see also discussion in Zahid et al. 2014). Therefore, strangulation does not have any visible (measurable) effect on metallicities of Mhigh objects.

The quenching (strangulation) is likely initiated when galaxies pass $R_{200}$, but this first only affects the gas metallicities and does not affect the SSFRs of cluster objects much (see Fig. 4). The galaxies then slowly use up their existing gas reservoir through star formation. However, because galaxies inside $R_{200}$ can travel to denser inner regions of the cluster in about $1 \mathrm{Gyr}$, it is probable that ram-pressure stripping processes are more pronounced with time, contributing to a rapid complete quenching of star formation at a later time, as we discuss in Sect. 4.4.

\subsection{Predictions of $f_{\mathrm{SF}}$ from simulations for different time delays between accretion and quenching}

To constrain the timescales on which star formation is quenched as galaxies fall into clusters, we measure how the observed fraction of star-forming galaxies increases with clustercentric radius (the star formation-density relation, see histograms in Fig. 3), and compare these values with predictions from cosmological simulations, adopting the same approach as Haines et al. (2015). They examined the spatial distributions and orbits of galaxies in the vicinity of the 75 most massive $\left(3-21 \times 10^{14} M_{\odot}\right)$ clusters from the Millennium simulation (Springel et al. 2005), a cosmological dark matter simulation covering a $\left(500 \mathrm{~h}^{-1} \mathrm{Mpc}\right)^{3}$ volume.

Twenty $\times 20 \times 140 \mathrm{~h}^{3} \mathrm{Mpc}^{3}$ volumes centered on each cluster are extracted and extended in the $z$-direction such that, for a distant observer viewing along this axis, all galaxies with line-of-sight velocities within $5000 \mathrm{kms}^{-1}$ of the cluster redshift are included, enabling projection effects to be fully accounted and quantified. Using a database with information on simulated galaxy properties and dark matter halos (see more details in Haines et al. 2015), we can follow the orbit of each galaxy with respect to the cluster from formation to the present day, enabling us to determine its epoch of accretion into the cluster defined as the redshift at which the galaxy passes within $R_{200}$ for the first time. The backsplash galaxies discussed below are still hosted by dark matter halos, so their positions and orbits are well defined, simply by tracking their host dark matter halo. An important point is that we take into account projection effects, since we are looking through the cluster, not at it. Thus we include all the galaxies along the line of sight, but which would be identified as spectroscopic cluster members since they are within the wider overdense region.

The stacked radial population gradients for 75 massive clusters from the Millennium simulation are reproduced, as they would appear if observed at $z \sim 0.2$, to best match the redshifts of the LoCuSS sample. The model galaxy positions and velocities relative to the cluster halo are measured as they stood at $z \sim 0.2$, and the clusters are stacked using their $R_{200}$ values measured at $z \sim 0.2$. The form of the black dashed curve in Fig. 7 is mostly due to the large population of galaxies in the wider overdensity around the cluster, which are still on their first infall but still outside $R_{200}$; these galaxies become increasingly important in terms of numbers for increasing clustercentric radius, combined with the backsplash galaxies whose orbits back out beyond $R_{200}$. In this simple toy model we assume that quenching is initiated when infalling field galaxies pass physical $R_{200}$ of the cluster for the first time, based on our observational results of enhanced metallicities at $R<R_{200}$ and initiated quenching at $R_{200}$ and also in agreement with Wetzel et al. (2012) who concluded that infalling massive galaxies experience no significant quenching of star formation until they arrive within $R_{200}$ of the more massive host halo. It should be noted that the observational (tentative) evidence for quenching initiated when galaxies pass $R_{200}$ for the first time is a new result from our analysis, and was not known in the work of Haines et al. (2015) who also considered models in which star formation starts to be quenched when galaxies pass $R_{500}$ or $2 R_{200}$ for the first time (their Figs. 14 and 15).

This onset of quenching could result either in the star formation of infalling galaxies being instantaneously completely quenched at the moment they pass physical $R_{200}$, or completely quenched after a time delay meanwhile slow (but not complete) quenching can already happen. Complete quenching means for our observational sample that galaxies leave the SFgals and enter the nonSFgals sample. To consistently compare the observed relation with the models, the fraction of star-forming galaxies $f_{\mathrm{SF}}$ versus clustercentric radius relation shown in Fig. 7 assumes that the population of infalling galaxies has the same $f_{\mathrm{SF}} \sim 40 \%$ as our observed field galaxy sample (blue solid lines in Fig. 7), i.e., $40 \%$ of field infalling galaxies are in the SFgals sample and $60 \%$ in the nonSFgals sample.

We note that this $f_{\mathrm{SF}}$ versus clustercentric radius diagram cannot distinguish between a delayed-then-rapid quenching and a slow-then-rapid quenching scenario. It just indicates how long galaxies remain classified as star-forming (SFgals) before they are quenched becoming nonSFgals. Their star formation may be hardly affected during this delay time or may be slowly declining while they remain classified as SFgals.

For $R_{200}<R<2 R_{200}$ the "instantaneous quenching" model $f_{\mathrm{SF}}$ curve (dashed line in Fig. 7) does not reach the field $f_{\mathrm{SF}}$ value of about $40 \%$ (as indicated by the area between the blue solid lines). This is because of the contribution of quenched backsplash galaxies that had been previously accreted into the cluster, reaching well within $R_{200}$ on first pericentric passage at earlier times and quenching star formation, but now have bounced back out beyond $R_{200}$ (e.g., Gill et al. 2005; Ludlow et al. 2009). We note that quenched backsplash galaxies appear as nonSFgals in our LoCuSS sample. The solid model curves show the effects of delaying the moment at which complete quenching occurs, 


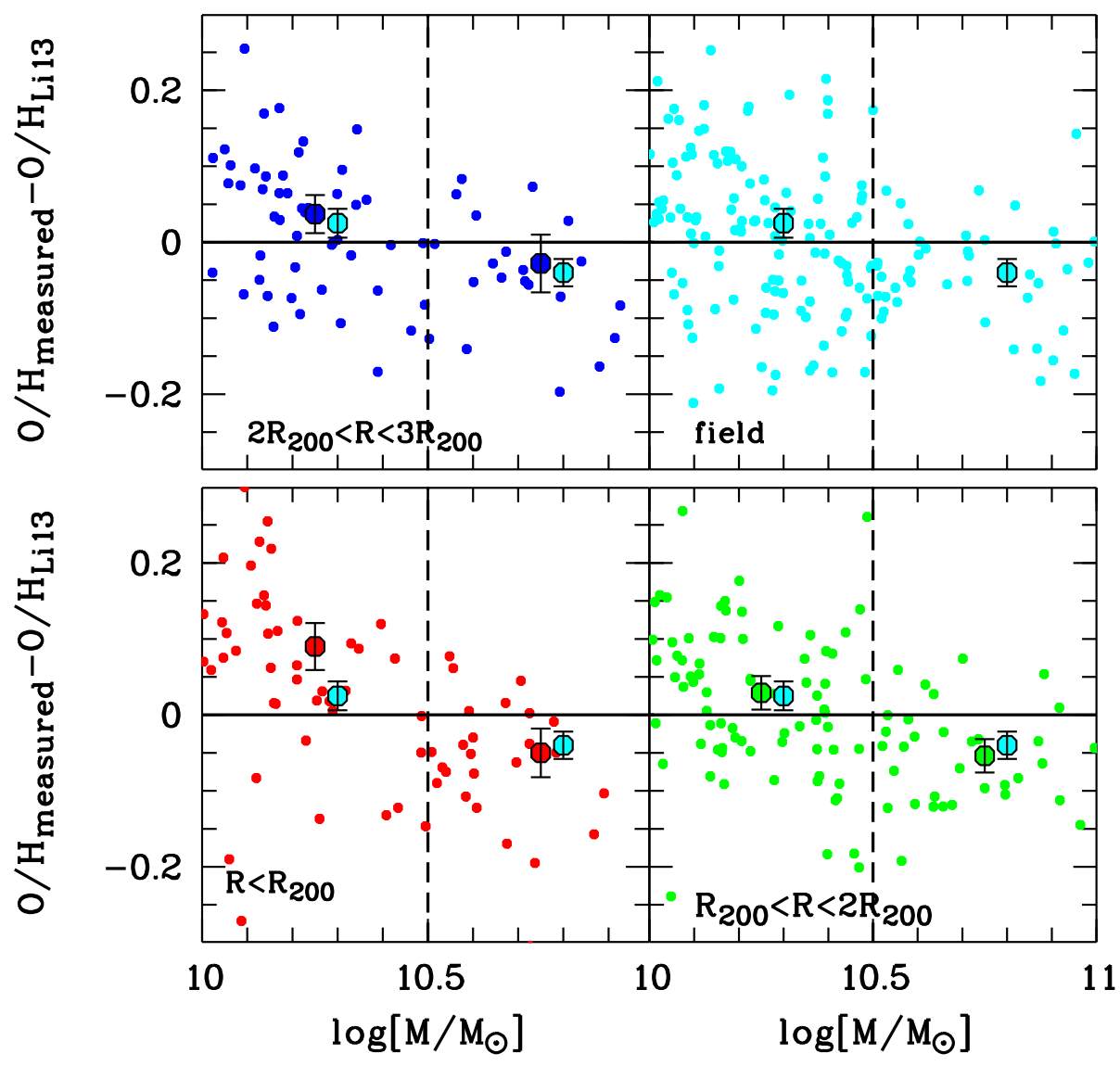

Fig. 6. Difference between the measured $\mathrm{O} / \mathrm{Hs}$ of $z \sim 0.2$ cluster galaxies and the expected $\mathrm{O} / \mathrm{Hs}$ from the formulations of Lilly et al. (2013) for three regions at different distances from the cluster center (red, green, and blue) and field galaxies (cyan). The median value of the differences in metallicities of Mlow cluster galaxies inside $R_{200}$ (large red filled circle) indicates that measured $\mathrm{O} / \mathrm{Hs}$ are higher inside $R_{200}$ compared to the Lilly et al. (2013) predictions with inflow of pristine gas. The median $\mathrm{O} / \mathrm{Hs}$ of Mlow field galaxies (large Mlow cyan filled circle) and the median $\mathrm{O} / \mathrm{Hs}$ of Mlow cluster galaxies at $R>R_{200}$ are quite consistent $(1.3 \sigma)$ with the predictions. This indicates that the environment has an influence on gas metallicities with slow quenching (strangulation) likely initiated when galaxies pass $R_{200}$. by terminating star formation only in those galaxies accreted into the cluster more than $\Delta t$ Gyr prior to observations, where $\Delta t$ corresponds to the delay time in the delayed-then-rapid quenching scenario of Wetzel et al. (2013). The red curve (1 Gyr delay between accretion and complete quenching) diverges from the dashed curve (instantaneous rapid quenching when accreted at $R_{200}$ ) only for $R<R_{200}$ because the most recently accreted galaxies (less than $1 \mathrm{Gyr}$ ago) have not had sufficient time to pass through the clusters and go back beyond $R_{200}$.

\subsection{Observed $f_{\mathrm{SF}}$ and implied quenching timescales}

The observed fraction $f_{\mathrm{SF}}$ of star-forming galaxies in our cluster and field samples was computed based on the classification as SFgals using spectroscopic $\mathrm{H} \alpha$ measurements (as described in Sect. 2.2); these measure instantaneous SFRs (timescale $<10 \mathrm{Myr}$ ), which are different from the LoCuSS classification based on $24 \mu \mathrm{m}$ SFRs by Haines et al. (2013, 2015). Thus, our SFgals sample is not a subsample of the sample used by Haines et al. $(2013,2015)$. The SFRs from $24 \mu \mathrm{m}$ have significant contributions from slightly older populations (as shown by, e.g., Salim et al. 2009) that have timescales over which the star formation is measured being effectively on the order of $100 \mathrm{Myr}$. Therefore, using $f_{\mathrm{SF}}$ based on $\mathrm{H} \alpha$ measurements we can measure quenching timescales with a complementary SFR indicator compared to $24 \mu \mathrm{m}$, which was used by Haines et al. (2015) to derive quenching timescales. Using $\mathrm{H} \alpha$ which measures SFRs on shorter timescales than $24 \mu \mathrm{m}$, we are able to detect the quenching of star formation after a shorter time (i.e., more accurate).

The likelihood that a given galaxy was targeted for spectroscopy depends strongly on both its location with respect to the cluster center as well as its photometric properties (e.g., $K$-band magnitude, $J-K$ color) as detailed in Haines et al. (2013). To account for this, each galaxy shown in the phasespace diagram and histograms in Fig. 3 is additionally weighted by the inverse probability of the galaxy having been observed spectroscopically, following the approach of Norberg et al. (2002). The resulting $f_{\mathrm{SF}}$ are shown as black filled circles in Fig. 7. Error bars for the observed fraction of star-forming galaxies $f_{\mathrm{SF}}$ are computed using Poisson statistics $\Delta f_{\mathrm{SF}}=f_{\mathrm{SF}}$. $\sqrt{1 / N_{\mathrm{SF}}+1 / N_{\mathrm{SFnonSF}}-2 / N_{\mathrm{SF}} \cdot N_{\mathrm{SFnonSF}}^{-1 / 2}}$, where $N_{\mathrm{SF}}$ is the number of star-forming galaxies and $N_{\mathrm{SFnonSF}}$ is the total number of star-forming and non-star-forming galaxies. The comparison of the observed $f_{\mathrm{SF}}$ (black symbols) with the model curves derived from the Millennium simulation implies quenching timescales of 1-2 Gyr, roughly consistent with a location between the red and cyan model curves, given the error bars. Much longer quenching time delays ( $\Delta t>3 \mathrm{Gyr}$, green curve) are clearly excluded because they would leave too many star-forming galaxies at $R<2 R_{500}$, which is not observed. These quenching time delays of 1-2 Gyr are consistent with the delay times for cluster galaxies with similar stellar masses $\left(10 \leq \log \left(M / M_{\odot}\right) \leq 11\right)$ found by Balogh et al. (2016), as shown in their Fig. 7, and also with the quenching timescales of 1-2 Gyr inferred by Haines et al. (2013, 2015) for galaxies accreted inside $R_{200}$.

\subsection{Slow -traced by enhanced metallicities - then rapid quenching}

Haines et al. (2013) found that massive star-forming galaxies are slowly quenched upon accretion into massive clusters. This is consistent with a gradual shut down of star formation in 


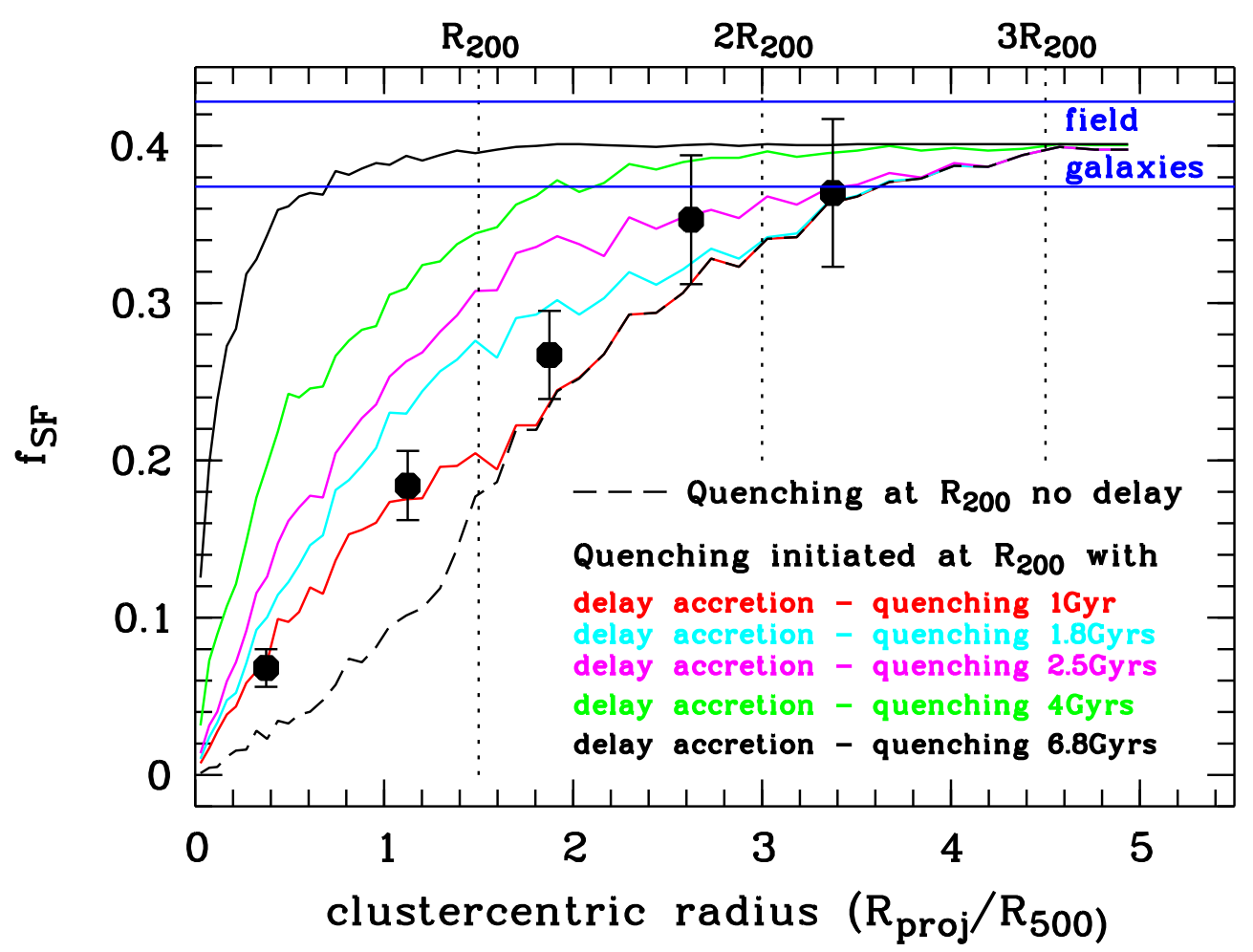

Fig. 7. Comparison of the observed fraction of star-forming galaxies $f_{\mathrm{SF}}$ as a function of clustercentric radius (based on the histograms shown in Fig. 3) with predictions from the Millennium cosmological simulation. The black filled circles show the observed fraction of star-forming cluster galaxies in the LoCuSS mass-complete sample. Each galaxy is weighted by the inverse probability of it having being observed spectroscopically, following the approach of Norberg et al. (2002). The black dashed line shows the predicted star formation-radius relation using 75 massive clusters in the Millennium simulation, assuming that star formation is immediately quenched upon being accreted by the cluster, i.e., passing the physical $R_{200}$ for the first time. The additional (colored) solid curves show the effects of delaying the complete quenching by a time $\Delta t$ after the galaxy is accreted into the cluster (i.e., passing $R_{200}$ for the first time), corresponding to the delayed-then-rapid quenching scenario of Wetzel et al. (2013) or to a slow-then-rapid model as discussed in Sect. 4.4. The comparison of the observed fractions with the model curves derived from the Millennium simulation implies $\Delta t \sim 1-2 \mathrm{Gyr}$.

infalling spiral galaxies as they interact with the ICM via rampressure stripping or strangulation mechanisms. The SFRs of these infalling galaxies were found to decline exponentially on quenching timescales in the range $0.7-2.0 \mathrm{Gyr}$.

On the other hand, Wetzel et al. (2013) concluded that the SFRs of satellite cluster galaxies evolve, at least on average, via a delayed-then-rapid quenching scenario. The SFRs of lower mass satellites remain unaffected for a delay of a few Gyr after first infall, and this delay timescale was claimed by Wetzel et al. (2013) to be shorter for more massive satellites. This is consistent with $\Delta t \sim 1-2$ Gyr we found for $10 \leq \log \left(M / M_{\odot}\right) \leq 11$ cluster galaxies (Fig. 7). After the delay phase, the SFRs of accreted satellite galaxies are expected to decline rapidly in the Wetzel et al. (2013) model.

This delayed-then-rapid quenching scenario is compatible with our findings if changed to a slow-the-rapid quenching scenario. There is a significant difference in the interpretation compared to Wetzel et al. (2013). Slow quenching (strangulation) is initiated when infalling galaxies first pass $R_{200}$ and is traced by an enhacement of $\mathrm{O} / \mathrm{Hs}$ of Mlow galaxies (due to the stopping of gas inflow) for a (delay) time $\Delta t \sim 1-2 \mathrm{Gyr}$.

Maier et al. (2006) constructed a large grid of Pégase 2 models to explore which region of the parameter space could reproduce the constraints imposed by the local metallicity-luminosity relation and by the metallicities and luminosities of galaxies at higher redshifts. They explored models in which the gas inflow on the model galaxy stops and the galaxy subsequently forms stars in a "closed-box"-like scenario. The tracks of these closed-box models show that after the gas inflow stops galaxies increase their metallicities by $\sim 0.2$ dex in $t_{\text {enrich }} \sim 1$ Gyr. Thus, strangulated infalling galaxies can increase their metallicities by $\sim 0.2$ dex while they move toward the central region of the cluster. This enrichment time is smaller than the typical crossing time $t_{\text {crossing }}=R_{200} / \sigma$ of our studied clusters of about 2 Gyr. During strangulation the inflow of new gas is cut out, but the cold interstellar medium disk is not directly perturbed. In this case the star formation can continue, using the gas available in the disk (resulting in higher gas-phase metallicities) until it is completely used up, unless an additional effect, before the gas is used up, quickly and completely quenches this star formation.

Our observational results revealing enhanced metallicities inside $R_{200}$ and the quenching timescales that we derive by comparing the observed $f_{\mathrm{SF}}$ with theoretical models and simulations in Fig. 7 can constrain and identify a likely physical quenching mechanism. This physical mechanism has to be active around $R_{200}$ to stop the infall of pristine gas on the galaxies. The galaxies then remain in the SFgals sample (see Fig. 4) in a slowquenching phase, while they travel to denser inner regions of the cluster. They slowly use up their existing molecular and HI gas through star formation over a period of about 2-3 Gyr, according to the constant molecular gas consumption timescales observed for the disks of nearby spiral galaxies spanning a wide range of properties (Bigiel et al. 2011) and based on the gas consumption timescales of star-forming galaxies derived from their 
measured HI and molecular gas masses in our studied LoCuSS A963 cluster (Jaffé et al. 2015; Cybulski et al. 2016). However, our derived quenching timescales of $1-2 \mathrm{Gyr}$ indicate that an additional effect has to quickly completely quench star formation before the existing gas is used up in 2-3Gyr, to move galaxies from the SFgals to the nonSFgals sample producing the observed $f_{\mathrm{SF}}$ versus clustercentric radius distribution shown in Fig. 7. The physical mechanism producing this fast quenching has to be active when galaxies arrive in denser inner regions of the clusters, where the density of the ICM is larger. This dependence of the quenching mechanism on the density of the ICM (which increases with decreasing clustercentric radius of the clusters) makes ram-pressure stripping a promising candidate for the slow-then-rapid quenching scenario, and we quantitatively explore this in the following.

Gas can be removed from infalling galaxies if the ram pressure exceeds the restoring force per unit area (gravitational restoring pressure) exerted by the galaxy, as first derived by Gunn \& Gott (1972). Using high-resolution cosmological hydrodynamic simulations, Bahé et al. (2013) computed the density of the ICM and derived the ram pressure $P_{\text {ram }}$ in clusters. For massive clusters $\left(\sim 10^{15} M_{\odot}\right)$ such as those we are studying, they estimated a range $P_{\text {ram }} \sim 3 \times 10^{-14} \mathrm{~N} \mathrm{~m}^{-2}-10^{-12} \mathrm{~N} \mathrm{~m}^{-2}$ near $R_{200}$ for $90 \%$ of the simulated galaxies (Fig. 9 in Bahé et al. 2013). On the other hand, the typical restoring pressure derived by Bahé et al. (2013) for massive galaxies $\left(10 \leq \log \left(M / M_{\odot}\right) \leq\right.$ 11) is $\sim 3 \times 10^{-12} \mathrm{~N} \mathrm{~m}^{-2}-10^{-11} \mathrm{~N} \mathrm{~m}^{-2}$ on cold gas and $\sim 3 \times$ $10^{-14} \mathrm{~N} \mathrm{~m}^{-2}$ on hot gas at a radial distance from the galaxy center enclosing $0.1 M_{*}$ in cold gas. The reason for this restoring pressure difference is that cold gas is denser and sits much closer to the galactic center.

Comparing the ram pressure $P_{\text {ram }}$ at $R_{200}$ with the restoring pressure, it is clear that the ram-pressure is too low at $R_{200}$ to strip cold gas, but becomes just sufficient $\left(>3 \times 10^{-14} \mathrm{~N} \mathrm{~m}^{2}\right.$ ) for most infalling galaxies to strip the more extended, less dense and less tightly bound hot gas. Thus, while not impacting star formation directly, the removal of hot gas at $R_{200}$ ends the replenishment (through cooling) of cold gas resulting in a delayed decrease in star formation as the remaining cold gas is consumed (strangulation). At a later time, meanwhile the galaxies travel to denser inner regions of the cluster, $P_{\text {ram }}$ increases to values $P_{\text {ram }}>3 \times 10^{-12} \mathrm{~N} \mathrm{~m}^{2}$ comparable to the restoring pressure of cold gas, sufficient to strip cold gas in massive cluster galaxies, producing a rapid phase of (complete) quenching of star formation.

This interpretation is also consistent with the MKZ16 results about strangulation traced by enhanced metallicities initiated at $\sim 2 R_{200}$ (Fig. 7 in MKZ16) for lower mass, $9 \leq \log \left(M / M_{\odot}\right) \leq$ 10 , galaxies in the massive $10^{15} M_{\odot}$ MACSJ0416.1-2403 cluster. At $2 R_{200}$ the ram pressure calculated by Bahé et al. (2013), $P_{\text {ram }}>3 \times 10^{-15} \mathrm{~N} \mathrm{~m}^{-2}$, becomes just comparable with the restoring pressure of the hot gas in the range $10^{-14} \mathrm{~N} \mathrm{~m}^{-2}-10^{-15} \mathrm{~N} \mathrm{~m}^{-2}$ of lower mass galaxies, resulting in stripping of their hot halo gas and initiating strangulation.

Steinhauser et al. (2016) used ram-pressure stripping simulations employing the moving-mesh code AREPO (Springel 2010) to follow at high resolution the interaction of a galaxy cluster with infalling galaxies. These authors constructed several different galaxy models, including variations of the amount of gas in the halos of the galaxies, and collided these models with galaxy clusters of different mass. In agreement with our observational results of an slow-then-rapid quenching in clusters, Steinhauser et al. (2016) found that, typically, their model galaxies continue to form stars with only slightly modified rates as a result of the stripping of the hot gaseous halos (strangulation) of the galaxies. On the other hand, the cold gas is stripped only during pericenter passages with small pericenter distances leading to a full quenching of star formation on a short timescale.

\section{Summary}

This study of environmental effects on the $Z\left(M_{*}, \mathrm{SFR}\right)$ relation is based on Hectospec spectroscopy of cluster galaxies in seven clusters from LoCuSS at $0.15<z<0.26$. The main results can be summarized as follows:

1. Cluster and field galaxies at $0.15<z<0.26$ show, on average, similar MZR and mass-SSFR relations for stellar masses $10 \leq \log \left(M / M_{\odot}\right) \leq 11$ (Figs. 4 and 5). There is, however, tentative evidence $(2.4 \sigma$ significance) that $10 \leq$ $\log \left(M / M_{\odot}\right)<10.5$ galaxies inside $R_{200}$ (red symbols and histograms in Fig. 5) have metallicities displaced to higher values compared to field counterparts and to cluster galaxies at $R>R_{200}$.

2. While the measured $\mathrm{O} / \mathrm{Hs}$ of field and $R>R_{200}$ cluster galaxies are in good agreement with FMR predictions, $10 \leq \log \left(M / M_{\odot}\right)<10.5$ cluster galaxies inside $R_{200}$ have a distribution shifted to higher metallicities than predicted by the Lilly et al. (2013) models with inflowing gas (Fig. 6), indicating that a strangulation scenario in which gas inflow has stopped producing enhanced metallicities is a plausible mechanism. For $10.5 \leq \log \left(M / M_{\odot}\right) \leq 11$ galaxies, the MZR saturates and becomes flat for cluster and field galaxies (Fig. 5). Therefore, strangulation does not have any visible effect on the metallicities of $10.5 \leq \log \left(M / M_{\odot}\right) \leq 11$ objects.

3. We derive quenching timescales $\Delta t=1-2 \mathrm{Gyr}$, defined as the delay time between the time the galaxies pass $R_{200}$ until complete quenching of star formation. This is carried out by comparing the fraction of star-forming cluster galaxies as a function of clustercentric radius to model curves derived from the Millenium simulation (Fig. 7). The model curves with the fraction of star-forming cluster galaxies as a function of clustercentric radius show the effects of delaying the moment at which the complete quenching occurs by terminating star formation only in those galaxies accreted into the cluster more than $\Delta t \mathrm{Gyr}$ prior to observations.

The main conclusion is that a strangulation scenario in a slowthen-rapid framework is plausible to explain the metallicities and SSFRs of field and cluster galaxies at different clustercentric radii, indicating that the inflow of gas is being stopped when cluster galaxies pass $R_{200}$, as a result of cluster environmental effects. This is likely due to the removal of the hot halo gas by ram-pressure effects. During the slow-quenching phase of $\Delta t=1-2$ Gyr these galaxies that are richer in metal inside $R_{200}$ are still star forming. After this $\Delta t$, meanwhile galaxies have traveled to denser inner regions of the cluster where the ram pressure also exceeds the gravitational restoring pressure of the cold gas, their star formation is likely completely quenched by ram-pressure stripping of their cold gas in the rapid phase.

Acknowledgements. This publication is supported by the Austrian Science Fund (FWF).

\section{References}

Bahé, Y. M., McCarthy, I. G., Balogh, M. L., \& Font, A. S. 2013, MNRAS, 430, 3017

Baldwin, J. A., Phillips, M. M., \& Terlevich, R. 1981, PASP, 93, 5 Balogh, M. L., McGee, S. L., Mok, A., et al. 2016, MNRAS, 456, 4364 
Bekki, K., Couch, W. J., \& Shioya, Y. 2002, ApJ, 577, 651

Bell, E. F., McIntosh, D. H., Katz, N., \& Weinberg, M. D. 2003, ApJSS, 149, 289

Bigiel, F., Leroy, A. K., Walter, F., et al. 2011, ApJ, 730, 13

Bresolin, F., Kudritzki, R.-P., Urbaneja, M. A., et al. 2016, ApJ, 830, 64

Chabrier, G. 2003, PASP, 115, 763

Cid Fernandes, R., Stasinska, G., Mateus, A., \& Vale Asari, N. 2011, MNRAS, 413, 1687

Cybulski, R., Yun, M. S., Erickson, N., et al. 2016, MNRAS, 459, 3287

Davé, R., Finlator, K., \& Oppenheimer, B. D. 2012, MNRAS, 421, 98

Davies, B., Kudritzki, R.-P., Lardo, C., et al. 2017, ApJ, 847, 112

Dayal, P., Ferrara, A., \& Dunlop, J. S. 2013, MNRAS, 430, 289

Dressler, A. 1980, ApJ, 236, 351

Elbaz, D., Daddi, E., Le Borgne, D., et al. 2007, A\&A, 468, 33

Ellison, S. L., Patton, D. R., Simard, L., \& McConnachie, A. W. 2008, ApJL, 672, 107

Gill, S. P. D., Knebe, A., \& Gibson, B. K. 2005, MNRAS, 356, 1327

Gordon, Y. A., Pimbblet, K. A., Owers, M. S., et al. 2018, MNRAS, 475, 4223

Gunn, J. E., \& Gott, J. R. I. 1972, ApJ, 176, 1

Haines, C. P., Pereira, M. J., Smith, G. P., et al. 2013, ApJ, 775, 126

Haines, C. P., Pereira, M. J., Smith, G. P., et al. 2015, ApJ, 806, 101

Jaffé, Y. L., Smith, R., Candlish, G. N., et al. 2015, MNRAS, 448, 1715

Kauffmann, G., Heckman, T. M., Tremonti, C., et al. 2003, MNRAS, 346, 1055

Kennicutt, Jr., R. C. 1998, ARA\&A, 36, 189

Kewley, L. J., et al. 2001, ApJS, 132, 37

Kewley, L. J., \& Dopita, M. A. 2002, ApJSS, 142, 35

Kewley, L. J., Dopita, M. A., Leitherer, C., et al. 2013, ApJ, 774, 100

Larson, R. B., Tinsley, B. M., \& Caldwell, C. N. 1980, ApJ, 237, 692

Lara-López, M. A., Cepa, J., Bongiovanni, A., et al. 2010, A\&A, 521, A53

Lilly, S. J., Carollo, C. M., Pipino, A., et al. 2013, ApJ, 772, 119

Ludlow, A. D., Navarro, J. F., Springel, V., et al. 2009, ApJ, 692, 931
Maier, C., Lilly, S., Carollo, C. M., et al. 2006, ApJ, 639, 858

Maier, C., Lilly, S. J., Ziegler, B. L., et al. 2014, ApJ, 792, 3

Maier, C., Ziegler, B. L., Lilly, S. J., et al. 2015, A\&A, 577, A14

Maier, C., Kuchner, U., Ziegler, B. L., et al. 2016, A\&A, 590, A108

Mannucci, F., Cresci, G., Maiolino, R., et al. 2010, MNRAS, 408, 2115

Mink, D. J., Wyatt, W. F., Caldwell, N., et al. 2007, in Astronomical Data Analysis Software and Systems XVI, eds. R. A. Shaw, F. Hill, \& D. J. Bell (San Francisco, CA: ASP), ASP Conf. Ser., 376, 249

Norberg, P., Cole, S., Baugh, C. M., et al. 2002, MNRAS, 336, 907

Peng, Y.-J., Lilly, S. J., Kovač, K., et al. 2010, ApJ, 721, 193

Peng, Y., Maiolino, R., \& Cochrane, R. 2015, Nature, 521, 192

Pereira, M., Egami, E., Haines, C., \& Hardegree-Ullman, E. 2012, AAS Meeting, 219, 223.04

Pettini, M., \& Pagel, E. J. 2004, MNRAS, 348, 59

Pozzetti, L., Bolzonella, M., Lamareille, F., et al. 2007, A\&A, 474, 443

Renzini, A., \& Peng, Y. 2015, ApJ, 801, 29

Salim, S., Rich, R. M., Charlot, S., et al. 2007, ApJS, 173, 267

Salim, S., Dickinson, M., Rich, R. M., et al. 2009, ApJ, 700, 161

Salpeter, E. E. 1955, ApJ, 121, 161

Smith, G. P., Khosroshahi, H. G., Dariush, A., et al. 2010, MNRAS, 409, 169

Springel, V. 2010, MNRAS, 401, 791

Springel, V., White, S. D. M., Jenkins, A., et al. 2005, Natur, 435, 629

Steinhauser, D., Schindler, S., \& Springel, V. 2016, A\&A, 591, A51

Tremonti, C. A., Heckman, T. M., Kauffmann, G., et al. 2004, ApJ, 613, 898

Wetzel, A. R., Tinker, J. L., \& Conroy, C. 2012, MNRAS, 424, 232

Wetzel, A. R., Tinker, J. L., Conroy, C., \& van den Bosch, F. C. 2013, MNRAS, 432,336

Zahid, H. J., Dima, G. I., Kudritzki, R.-P., et al. 2014, ApJ, 791, 130

Zibetti, S., Charlot, S., \& Rix, H.-W. 2009, MNRAS, 400, 1181 